\title{
Norois
}

Environnement, aménagement, société

\section{Un label patrimonial pour des valeurs environnementales en France rurale : vers un capital environnemental?}

Heritage-branding and environmental values in rural France: For an environmental capital?

\section{Hélène Ducros}

\section{OpenEdition \\ Journals}

Édition électronique

URL : http://journals.openedition.org/norois/6122

DOI : 10.4000/norois.6122

ISBN : 978-2-7535-6537-1

ISSN : 1760-8546

Éditeur

Presses universitaires de Rennes

\section{Édition imprimée}

Date de publication : 2 novembre 2017

Pagination : 17-40

ISBN : 978-2-7535-6465-7

ISSN : 0029-182X

Référence électronique

Hélène Ducros, « Un label patrimonial pour des valeurs environnementales en France rurale : vers un capital environnemental ? », Norois [En ligne], 243 | 2017, mis en ligne le 20 septembre 2019, consulté le 15 mars 2021. URL : http://journals.openedition.org/norois/6122 ; DOI : https://doi.org/10.4000/ norois.6122 


Presses
Universilaires
de Rennes
www.pur-editions.fr
Revue en ligne : http://norois.revues.org

\title{
Un label patrimonial pour des valeurs environnementales en France rurale : vers un capital environnemental?
}

\author{
Heritage-branding and Environmental Values in Rural France: \\ For an Environmental Capital?
}

\author{
Hélène Ducros
}

University of North Carolina Wilmington, 601 S College Rd, Wilmington, NC 28403 (USA), (helenegeog@gmail.com)

\begin{abstract}
Résumé : En se penchant sur l'Association des Plus Beaux Villages de France, cet article considère les moyens par lesquels un modèle de mise en marque des territoires en France rurale produit et standardise des représentations paysagères basées sur des valeurs environnementales définies et véhiculées par des outils d'évaluation et d'exceptionnalisation. L'expérience des villages qui s'engagent dans cette forme de préservation du patrimoine dans leur projet de revitalisation rurale montre que non seulement l'Association expertise ces villages selon des canons environnementaux qu'elle perpétue en tant que représentatifs de paysages ruraux en France, mais ce faisant elle s'appuie sur des formes diverses de capital. Ceci complexifie la relation entre labellisation et environnement, venant ainsi flouter les contours d'un capital environnemental en devenir, tout en le validant.
\end{abstract}

\begin{abstract}
The examination of the Association of the Most Beautiful Villages of France delves into how a territorial branding model in rural France produces and standardizes certain landscape representations based on the label's environmental values, which are defined and circulated through localization and evaluation tools. The experience of localities that abide by the agenda of rural revitalization through heritage preservation reveals that the Association not only appraises whether villages embody the environmental canons it has delineated as representative of rural landscapes in France, but also that it rests on diverse forms of capital that entangle the relationship between labelization and environment, thus validating a budding concept of environmental capital while also blurring its boundaries.
\end{abstract}

Mots clés : labellisation - environnement - village - patrimoine rural - Association des Plus Beaux Villages de France

Keywords: branding - environment - village - rural heritage - Association of the Most Beautiful Villages of France

\section{INTRODUCTION}

En 2015, GEOLAB organisait à Limoges le colloque « Capital environnemental : représentations, pratiques, dominations, appropriations spatiales » afin d'interroger la valeur épistémologique d'un «capital environnemental » pour l'analyse des rapports société-milieu en géographie. GEOLAB en procure une proto-définition pour guider ceux souhaitant l'appliquer, l'enrichir ou la tester. Cet article s'inscrit dans le sillage de cette définition qui envisage le nouveau capital comme une grille d'analyse heuristique pour lire des objets d'étude spécifiques. Cette « clé d'interprétation » comprend les inves- 
tissements dans l'environnement effectués par des acteurs selon des représentations, intérêts, et systèmes de valeurs propres, mettant en évidence des rapports asymétriques ainsi que des pratiques et imaginations diverses. De plus, il est suggéré qu'un capital social, culturel ou symbolique pourrait se convertir en capital environnemental. Ou à l'inverse, un capital environnemental lui-même se convertirait en une autre forme de capital (GEOLAB, 2015). Placé dans l'héritage bourdieusien du capital (culturel, économique, social, ou symbolique) qui repose sur la nécessité d'un « champ » associé (Bourdieu, $1979 ; 2000$ ), i.e. un espace social structuré (Neveu, 2013), ce nouveau capital permettrait ainsi d'interroger le processus de création de valeurs environnementales, la mobilisation des acteurs qui les créent et les diffusent, ainsi que les relations société-environnement sur lesquelles elles s'appuient. À travers une approche empirique, on examine le potentiel explicatif d'un tel capital en élucidant la construction du champ environnemental à partir de valeurs. On envisagera aussi la validation théorique de ce capital quant à sa convertibilité réciproque avec d'autres formes de capital.

Puisque la relation entre ruralité et environnement n'est plus à démontrer, l'ancrage territorial étant au cœur des politiques contemporaines de gestion environnementale des espaces (Larrue, 2017), le cas concret présenté ici se penche sur la production et l'utilisation de valeurs environnementales au sein de communes rurales. En particulier, son but est de mieux comprendre une relation éventuelle entre patrimoine, ressources territoriales et environnement afin d'évaluer ce que le phénomène de labellisation territoriale pourrait apporter à l'élaboration d'un champ social de l'environnement et donc à la définition encore en devenir d'un «capital environnemental ». Compte-tenu que le patrimoine est devenu un soutien essentiel dans les projets locaux en milieu rural (Landel et Senil, 2009; Husson, 2008; Bonerandi, 2005), à partir de quelles réflexions, de quels éléments, et selon quel fonctionnement s'est constituée une association amenée à attribuer à des villages sélectionnés le label des Plus beaux villages de France (PBVF)? La labellisation territoriale est envisagée dans une problématique plus générale portant sur la préservation du patrimoine, ici rural, et les moyens de valorisation d'un patrimoine local par certains acteurs. Le concept de capital environnemental peut-il éclairer ces thématiques? Vice-versa, quels enseignements peuventils être tirés de la mise en marque des territoires patrimonialisés pour définir un capital environnemental comme cadre d'analyse des dynamiques de développement territorial ? Cette étude explore donc la relation potentielle entre un concept de capital environnemental et la labellisation des territoires à l'intersection de valeurs environnementales et de valeurs patrimoniales du lieu, tel que celui-ci est vécu, perçu, conceptualisé et imaginé.

\section{LABELLISATION TERRITORIALE, ENVIRONNEMENT, VALEURS}

\section{Label et patrimoine}

La labellisation territoriale s'est accélérée depuis la fin du Xx $x^{\text {e }}$ siècle (Fournier, 2014), désormais enrôlée dans les politiques de dynamisation territoriale (Filloz et Colomb, 2011). Cependant, on peine à théoriser l'application au lieu et à l'espace de concepts élaborés pour signifier ou certifier des biens et objets (Pike, 2015). Le film d'animation Logorama (Alaux, de Crécy et Houplain, 2009) témoigne de l'omniprésence des logos dans les représentations spatiales et la vie quotidienne. En effet, ils essaiment dans les paysages le long des routes et chemins de campagne: "Petites cités de caractère ", «Plus beaux villages de France », «Station verte», «Village patrimoine », "Villes et villages fleuris », ou encore "Patrimoine mondial », pour ne citer que quelques labels reconnus par touristes, acteurs locaux, habitants des lieux, ou spécialistes du patrimoine. En tant qu' « étiquettes », ces certifications sont de plus en plus souvent plébiscitées et fièrement arborées à l'entrée des communes dans leur quête de reconnaissance politique, économique, historique et culturelle, et ce à une échelle locale ou nationale, voire internationale. Généralement, ces labels se fondent sur une approche esthétique et/ou éthique du lieu. Ils mobilisent des citoyens et acteurs localement, même s'ils s'inscrivent souvent dans un cadre législatif et étatique plus large.

Le label déclenche nombre de questions sur les processus et les acteurs impliqués dans la valorisation de la ressource territoriale et paysagère dans le cadre de politiques locales constamment tiraillées entre développement productiviste et intérêt patri- 
monial (Landel et Senil, 2009). Le tourisme et le patrimoine sont alliés dans la production des territoires et leur aménagement (Lazzarotti, 2011), tandis que le paysage est le moyen par lequel les touristes prennent contact avec le lieu (Rickly-Boyd et al., 2014). De plus, l'élargissement du champ patrimonial, phénomène post-moderne (Wood, 1999), donne le jour au territoire-patrimoine ainsi qu'au paysage-patrimoine (Poulot, 2006). La nature ellemême fournit de nouveaux objets de patrimonialisation (Bouisset et Degrémont, 2013). Poussée jusqu’à «saturation» (Lowenthal, 2015), cette gloutonnerie patrimoniale repose sur un élan conservationniste fanatique dénoncé comme "dangereuse épidémie » et même « cancer métastatique » (id., citant l'architecte Koolhaas, p. 588) frappant des sociétés déracinées, inquiètes et obsédées de mémoire (Hartog, 2003) où tout objet est insufflé de valeur pour se patrimonialiser, y compris l'environnement.

\section{Environnement : nature-culture}

Débattre de la labellisation territoriale, c'est revenir sur la notion d'environnement. Certes, il représente un enjeu majeur de nos sociétés, se positionnant au cœur des politiques économiques et sociales contemporaines. Mais, tout en opérant un rapprochement entre le naturel et le social (Charbonnier, 2015), il n'appelle guère de définition traversant toutes les disciplines. D'autres concepts tels que paysage, milieu de vie, territoire, écosystème, lieu et espace - eux-mêmes constamment remaniés - compliquent la délinéation du terme. En particulier, la notion de paysage, difficile parfois à démarquer du terme environnement, est souvent utilisée par les acteurs de façon interchangeable, dans le sens du surrounding anglophone - ce qui nous entoure -. Alors que l'environnement est parfois réduit à sa dimension physique et naturelle, une appréhension holistique du paysage englobe plus adéquatement non seulement le milieu concret et objectif (souvent sa dimension naturelle résultant de l'observation), mais aussi le vécu - dimension expérientielle de la géographie Tuanienne (1974) - ainsi que le perçu, interprété à travers nos expériences passées - terrain de prédilection du géographe Meinig (1979) - et l'imaginélle rêvé (l'idée que l'on s'en fait). Ainsi, l'environnement, envisagé dans ses multiples dimensions dont celle de l'expérience directe, découle de la géographie du quotidien, de l'immersion des humains dans leur monde géographique, et de la totalité des relations directes qu'ils entretiennent avec celui-ci, comme le rappelle Seamon (1979) dans son analyse phénoménologique du mouvement, du repos et de la rencontre. Ces dimensions ne sont d'ailleurs pas forcément en harmonie les unes avec les autres dans la relation des individus avec leur environnement (Lowenthal et Riel, 1972).

D'autres facteurs perturbent l'appréhension de l'environnement. Interviennent différentes échelles spatiales (de la maison à la région ou nation, et jusqu'au translocal); différents chronotopes (configurations space-time spécifiques); et milieux mémoriels différenciés. De plus, l'environnement est à la fois social et personnel. Ces attributs le rendent difficile à fixer de façon à contenter toutes les disciplines, toutes les approches, et tous les acteurs, et cela avant même de l'associer à la notion de capital. En plus de ces éléments, on retient que l'environnement est une construction inclusive et relationnelle, i.e. une relation de co-dépendance intégrant individus et lieux dans leur réalité biophysique et représentations culturelles et politiques. Cette construction sociale repose sur des valeurs, dont des valeurs esthétiques font partie (Berleant, 1997). En outre, Richard (2010) cerne la « composante environnementale » en termes de paysages, de patrimoine bâti et de qualité sanitaire de l'environnement. Ainsi « le capital environnemental des espaces concernés, à travers la "nature", les paysages ou le patrimoine bâti joue un rôle fondamental dans les stratégies d'installation et d'appropriation des territoires... » (Guyot et Richard, 2009). Ce lien explicite entre environnement et paysage patrimonial appelle à s'interroger sur la conception de l'environnement que les acteurs privilégient et perpétuent.

\section{Capitaux pluriels et valeurs environnementales}

Alors que le capital environnemental émergerait comme une nouvelle conceptualisation théorique, le concept voisin de capital territorial est éprouvé, notamment retenu au sein de l'Union européenne et la politique de l'OCDE. Dans une optique de développement économique régional, il représente tous les biens matériels et immatériels, privés ou publics ayant une influence sur la performance économique 
(Capello et al., 2011) ou le système des assets territoriaux de nature économique, culturelle, sociale et environnementale qui assurent les lieux d'un développement potentiel (Perucca, 2014). D'autres déclinaisons du capital brouillent la définition d'un capital environnemental, tel le capital paysager (Cailly et Dodier, 2007), « landscape capital » ou « landesque capital » (Håkansson et Widgren, 2014), de même que gêne la tension entretenue avec d'autres formes de capitaux et son alliance ou assujettissement potentiels à ceux-ci (GEOLAB, 2015).

Au centre de ces thématiques se trouvent des valeurs et des acteurs, puisque "l'investissement dans le capital environnemental se fait au nom de valeurs environnementales construites socialement, variées et parfois contradictoires » (GEOLAB, 2015). Pareillement, dans le cadre des critères de la patrimonialisation, "les évaluations produites par les acteurs ("c'est beau”) s'opèrent au nom de valeurs (la beauté) qui elles-mêmes ressortissent à des catégories plus générales, des "registres de valeurs” »(Heinich, 2009). C'est la construction et utilisation de ces valeurs, à la fois environnementales et patrimoniales, que cette étude sur la labellisation en France rurale tente d'éclaircir, ainsi que la hiérarchie des acteurs qui les incarnent, les véhiculent, les diffusent et les convertissent en un capital qui peut être mobilisé à des fins diverses. Les valeurs environnementales avancées par GEOLAB (2015) pour définir le champ environnemental nécessaire à l'émergence d'un capital ainsi qualifié sont en effet toutes au cœur du processus de labellisation patrimoniale : habitat, qualité paysagère, pratique récréative, composante productive, et qualité environnementale et écologique. L'examen de la relation entre valeurs environnementales et labellisation territoriale à travers les $P B V F$ déterminera si d'autres qualités s'ajoutent à ce champ.

\section{L'ASSOCIATION DES PLUS BEAUX VILLAGES DE FRANCE : PRODUIRE, DÉFENDRE ET DIFFUSER DES VALEURS ENVIRONNEMENTALES}

\section{Terrain et méthodologie}

Emblématique de la labellisation territoriale en France, le label des PBVF a su, depuis 1982, marquer les paysages. S'il n'est pas le premier logo à être apparu aux entrées des communes rurales, il est le premier label à s'être imposé comme un incontournable à l'échelle nationale et même internationale. Association de référence, elle provoque maintes couvertures médiatiques dans la presse régionale et nationale, suscitant l'intérêt des médias audiovisuels à large audience, comme en témoignent les programmes leur ayant été consacrés tant sur les chaînes de télévision publiques que privées. Sans compter les nombreux membres du réseau Plus beaux villages de France (jusqu’à $91 \%$ de la sélection) médiatisés avec d'autres par l'émission Le village préféré des Français, et dont plusieurs sont sortis gagnants. De plus, l'Association est désormais sollicitée par la classe académique, comme dans le cas d'un récent colloque "Les labels dans le domaine du patrimoine » (université d'Orléans, décembre 2106) pour lequel les organisateurs l'ont appelée à intervenir. Ainsi c'est à travers celle-ci que cette étude appréhende la labellisation et le jeu des acteurs dans la patrimonialisation des lieux.

Cet article résulte d'un travail ethnographique effectué entre 2011 et 2014, après des visites exploratoires dans des villages du nord au sud de la France en toutes saisons. Trois niveaux d'entretiens $(\mathrm{n}=100+)$ ont permis de saisir le rôle et le ressenti des principaux acteurs : organisations (offices du tourisme, associations, partenaires institutionnels et financiers), maires et/ou conseillers municipaux de 22 communes labellisées, et habitants. Une étude plus approfondie fut menée dans cinq communes dans quatre régions (Auvergne, Provence, Touraine, et Dom-Tom), sélectionnées car offrant un cadre varié en matière d'attrait et de fréquentation touristiques, d'isolement géographique, et de contexte économique. Les participants furent sélectionnés selon les méthodes décrites par Patton (2002) : d'abord par maximum variation sampling afin d'obtenir un échantillon hétérogène tenant compte des différentes fonctions au sein du village, durées de résidence, et âges; puis dans un second temps par la méthode boule de neige pour élargir l'échantillon. Enfin, grâce à la générosité de l'association, l'enquête fut complétée par une observation participante au cœur de ses réunions et activités de sélection, telles les visites d'expertise sur site en vue de classement. Le protocole des entretiens, obligatoirement revu par le Institutional Review Board à l'université de Caroline du Nord (USA), 
fut approuvé et autorisé sur la base essentielle de la confidentialité et l'anonymat des personnes interrogées dont l'identité se doit d'être protégée, en particulier car l'on se trouve dans de petites communautés où tout le monde se connaît. Les réponses furent donc traitées dans leur ensemble jusqu'à saturation. Aussi, les images ${ }^{1}$ et les citations de propos personnels sont incluses ici quand elles exemplifient une réponse-type, sans qu'un auteur ou un village n'aient à être désignés.

Après 35 ans d'actions menées par les maires de communes rurales qui la dirigent par le biais de trois commissions (Qualité, Notoriété, Développement) et un nombre restreint de salariés spécialisés, l'association comprend aujourd'hui 156 communes labellisées. En postulant au réseau, des communes se soumettent volontairement à ses exigeants critères de sélection car elles voient en ce label une possibilité de faire revivre le village, d'y faire rester et venir des habitants, et de protéger et transmettre un patrimoine rural aux futures générations. Trois critères sont éliminatoires. La commune doit être de dimension rurale pour l'agglomération bâtie (bourg). Elle doit détenir un patrimoine architectural ou naturel confirmé par un minimum de deux périmètres de protection (site ou monument historique classé ou inscrit). Enfin, une délibération du conseil municipal doit appuyer la demande écrite de candidature. L'association estime le potentiel de candidatures à 6000 communes. Souvent sans succès, de nombreuses communes se sont soumises à une évaluation-qualité rigoureuse dans le but d'intégrer les rangs des villages « classés ». En effet, $82 \%$ des demandes ont échoué depuis 1991, date à laquelle l'Association se dote d'un document clé pour solidifier, professionnaliser et crédibiliser son action : la Charte de qualité.

\section{La Charte de qualité : des critères " objectifs "}

Depuis 1991, la démarche de labellisation repose sur cette pièce maîtresse au sein de l'expertise. La Charte, avec sa grille de 27 critères, prescrit les atouts nécessaires conditionnant l'entrée dans le cercle fermé des villages classés. Elle suggère une vision holistique du paysage rural basée sur la réa-

1. Toutes les photos ont été prises par l'auteur entre 2011 et 2014. lité concrète de sa richesse patrimoniale mais aussi sur les possibilités de transformations socio-économiques qu'il offre et la preuve d'une volonté politique locale pérennisée et partagée (par exemple par l'existence d'un document d'urbanisme et la participation à une intercommunalité). La Charte est mobilisée au cours des délibérations de la Commission Qualité. Sur présentation du dossier établi par l'expert au cours de sa visite sur site, celleci décide de l'intégration de nouveaux membres, ou du maintien du label dans le cas des ré-expertises périodiques auxquelles sont soumis les villagesmembres tous les 6 ans.

Le «diagnostic » est établi selon des éléments esthétiques du bâti, mais aussi paysagers, organisationnels, ainsi que selon l'engagement municipal dans la mise en valeur du lieu. La grille de critères dits « objectifs » permet « de mesurer l'importance et la valorisation du patrimoine du village, sa qualité architecturale, urbanistique et environnementale ainsi que la pertinence des actions de la commune en termes de maîtrise et de mise en valeur de son territoire (outils d'urbanisme, maîtrise des flux de fréquentation, aménagements esthétiques...) » (PBVF, web). Les critères associent plusieurs dimensions : la mise en valeur du lieu et du patrimoine local, le développement, la promotion et l'efficacité des outils dont s'est dotée la commune pour y mieux parvenir, ainsi que les activités d'animation mises en œuvre (voir encadré). Non seulement la Charte sanctionne la qualité architecturale de l'habitat et la qualité écologique et paysagère du lieu, mais elle sert aussi d'outil d'évaluation pour le potentiel récréatif (festivals, expositions, fêtes de rue) et d'accueil. Elle s'attarde sur les infrastructures facilitant la « consommation » du lieu par les visiteurs et sa mise en tourisme, mais aussi l'accessibilité, qu'elle soit physique, intellectuelle ou émotionnelle. Le patrimoine doit être déchiffré, interprété, expliqué afin d'être rendu lisible et compréhensible. Des parcours de visite sont conseillés, des panneaux explicatifs sont postés à des endroits stratégiques et des cartes sont souvent disponibles. Elle récompense une qualité « anti-moderniste » apparente du lieu, comme l'enfouissement des réseaux. Le label préconise de bonnes pratiques en matière de végétalisation, préférant les plantations d'essences locales de pleine terre (figures la-c - planche I) plutôt que les jardinières de géraniums (figure $1 \mathrm{~d}$ - planche $\mathbf{I}$ ), d'éclairage 
«de bon goût et d'une sobriété adaptée au lieu; pas de tubes néons multicolores en plein Bourbonnais ", et de matériaux de construction "nobles » afin de favoriser l'harmonie esthétique des volumes, des matières et des couleurs. "Nous assumons que nous préférons... des matériaux qui vivent, qui évoluent, qui se façonnent avec le temps, et même qui racontent une histoire, plutôt que le PVC» (membre Commission Qualité, 2012). Enfin, tout est fait de sorte que le village se maintienne «dans son jus », un leitmotiv maintes fois entendu sur le terrain. "Un plus beau village, c'est un village qui a su rester dans son jus. Éviter les erreurs et promouvoir un développement qui ait un sens par rapport à notre histoire, c'est ça le challenge » (un maire, 2013).

Souvent, une qualité paysagère déjà appréciée existe de facto dans les villages candidats (villages perchés, villages de bord de rivière). Cependant, l'esprit des lieux ou genius loci, i.e. les particularités dont les lieux tirent leur essence (Lowenthal, 2015), qui incluent apparence physique, activités sociales, et le sens qu'un vécu passé leur communique (Relph, 1976), est mis en valeur par une habile et subtile manipulation paysagère : par exemple, par la création ou amplification de certains cônes de vue qui permettront d'embrasser un plus large champ visuel (figure 2 - planche II), en coupant des arbres pour ouvrir une perspective, ou mettant en évidence certaines images phare de «typicité » locale multi-sensorielle. Le champ de lavande à l'entrée d'un village du sud répondra à l'attente du public, renforçant l'esprit du lieu en même temps qu'il forme une esthétique environnementale, non seulement visuelle mais olfactive (figure 3 - planche II). Le maire encouragera donc le propriétaire à reprendre un champ de lavandin abandonné. La qualité architecturale de l'habitat repose, elle, sur l'évaluation du patrimoine bâti : volumes, couleurs et formes doivent former un tout harmonieux (figure 4 - planche III). Des palettes de couleurs sont conseillées aux habitants (par le biais des mairies) pour les boiseries et façades, et les permis de construire sont attribués par les élus avec la Charte de qualité en filigrane, bien que l'Architecte des Bâtiments de France soit sollicité pour les périmètres de protection obligatoires (500 m autour d'un objet inscrit ou classé). Les maires s'abritent parfois derrière la Charte: «La Charte de qualité m'aide à faire comprendre aux gens pourquoi je ne peux pas attribuer le permis. Si je dis non comme ça, ils discutent. Si je dis non par rapport au label, ça les fait réfléchir, car malgré

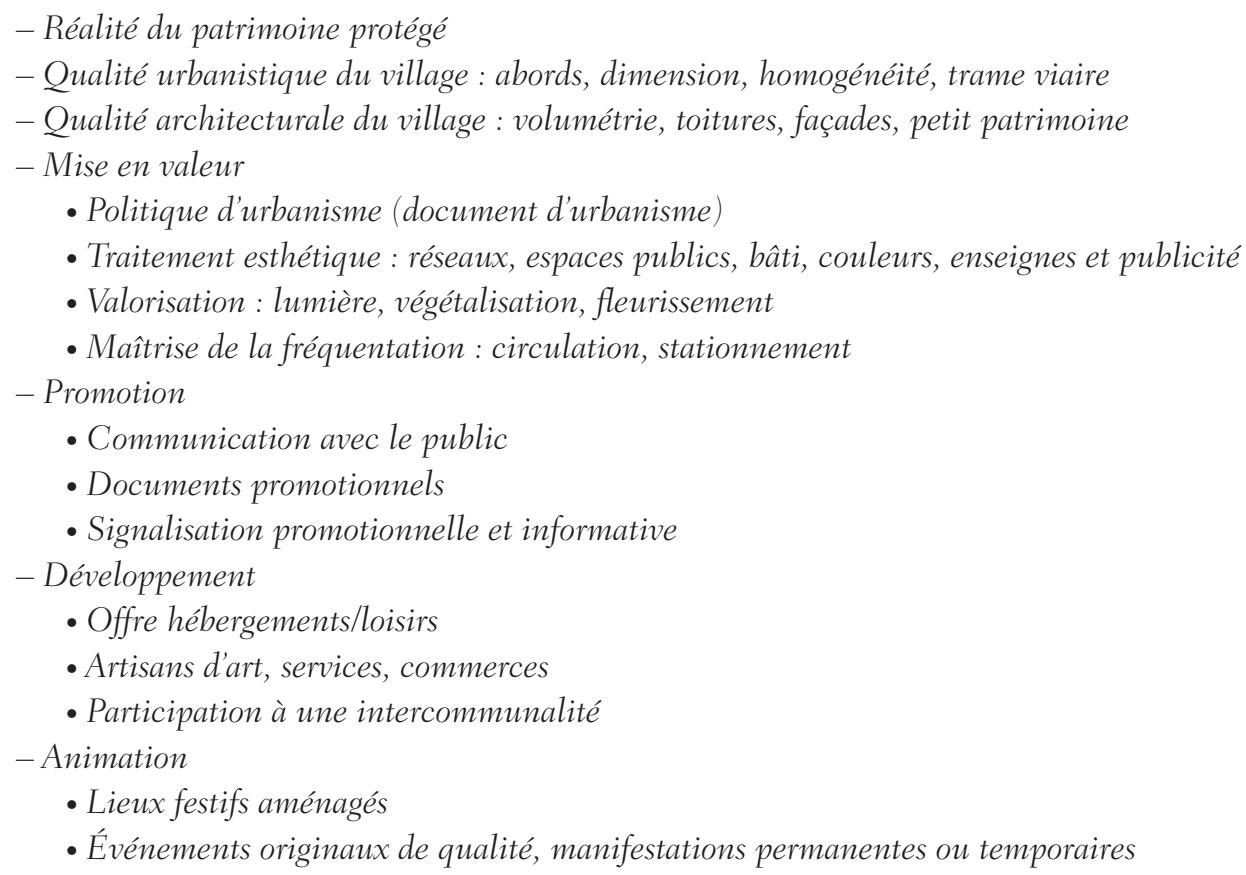


tout ils sont fiers et ils y tiennent au label. C'est leur village, c'est là qu'ils vivent. »

\section{LABEL PATRIMONIAL : PRÉSERVATION, ÉMOTION ET RÊVE}

\section{Des valeurs environnementales au service de la préservation du patrimoine}

Épicentre de l'association, la Charte assoit son idéologie patrimoniale et environnementale au-delà de la simple évaluation du bâti. Elle comprend également des critères d'évaluation pour les abords du bourg, l'expérience du lieu commençant déjà sur la route (figures 5 et 6 - planches IV-V), ainsi que les efforts à maintenir leur qualité esthétique et patrimoniale malgré des aménagements parfois nécessaires à la vie de la commune. Par exemple, on appréciera une arrivée sur le village qui met en évidence son implantation dans un paysage rural désormais patrimonialisé. «Les champs dans la côte... c'est la France. C'est ce qui reste de nos paysans. Un seul agriculteur ici, sur toute la commune. Il faut qu'il continue sinon le village disparâ̂tra derrière un écran de forêt. » Pour créer des logements, on préfère une greffe architecturale en osmose avec le patrimoine bâti en place, plutôt que d'avoir à traverser une aire pavillonnaire en pénétrant dans le centre historique. Alors que la résurrection de la vie de village est un objectif phare de l'Association, il ne s'agit pas de n'importe quelle vie. On saluera l'installation d'une activité commerciale en adéquation avec l'esprit du village et qui servira les habitants avant les touristes: "Peut-on acheter une baguette dans un PBV? »; «On ne veut pas que nos villages soient Las Vegas avec ses marchands du temple ", "ni une boutique de musée ». La Charte de qualité véhicule aussi des valeurs quant à la préservation du patrimoine vernaculaire, le « petit » patrimoine étant aujourd'hui très apprécié et valorisé : un pèse-bestiaux, un banc napoléonien, un lavoir inutilisé, un puits, ou une ancienne plaque émaillée... (figure 7 - planche VI). Elle valide également des valeurs mémorielles, paysagères et sociales du lieu souvent fondées sur un rapport à la nature : activités agricoles révolues, et jusqu'au souvenir d'un évènement douloureux comme une crue. « La crue - c'est quelque chose de dur, la nature -, on s'en souvient même si on n'était pas né. C'est bien qu'ils l'aient marquée. Il faut le dire aux autres qui ne savent pas" (figure 8 - planche VII).

\section{Un capital émotionnel : du lieu d'émotion au rêve}

Au-delà de l'appréciation d'un capital esthétique de l'environnement, c'est aussi l'évaluation de ce que les anglo-saxons appellent quality of life capital qui engage la Charte de qualité. Le but premier de l'Association étant de sortir les communes rurales de l'oubli, de l'isolement et de la stagnation afin de redonner vie aux campagnes en les valorisant, freinant ainsi un exode rural et peut-être même attirant de nouveaux venus, elle souligne que les habitants sont la cible principale de son action. Le tourisme devient un outil plutôt qu'une finalité. La Charte est explicitement mise au service de lieux d'émotions où l'on peut s'évader. Les commentaires ci-dessous expriment comment les acteurs internalisent cette notion, fussent-ils élus, experts au sein de l'association, ou habitants.

"Un plus beau village de France, on doit pouvoir le ressentir » (Un maire, 2012).

"Notre mission, c'est de créer des lieux d'émotions où l'on puisse se perdre »(PBVF, 2012).

"Quand on arrive dans un Plus beau village de France, on le sait, il y a quelque chose d'invisible, et pourtant c'est bien là. C'est comme... un air du passé qui vous fouette en pleine poitrine » (Habitant permanent, 2012).

À travers des critères dits « objectifs ", c'est donc paradoxalement un capital émotionnel basé sur le subjectif et la richesse affective qui émerge, et qui est en outre recruté par l'association dans l'élaboration de nouveaux axes de développement pour lesquels le faire rêver (PBVF, 2016) prolonge désormais la volonté délibérée de créer l'émotion.

\section{Label patrimonial : singulariser le lieu, uniformiser la marque}

Le label intervient dans la discussion sur le patrimoine environnemental en tant que garantie d'expérience multi-sensorielle. Il véhicule des valeurs patrimoniales et environnementales (articulées dans la Charte de qualité), et ainsi influence la relation spécifique de chaque acteur avec son patrimoine et environnement. Mais le label, en tant que promesse d'excellence, contrat ou garantie, doit aussi protéger 
son image, car il acquiert sa propre identité. Il doit préserver sa réputation au nom des valeurs qui le guident et qu'il crée. Labels et marques sont en effet des outils de communication, mais aussi le reflet d'une introspection identitaire des acteurs visant à établir une relation de confiance multipartite afin de fidéliser ceux qui adhèrent aux valeurs défendues, que ceux-ci soient de passage, des décideurs ou des habitants. Ainsi un objectif central du label est de créer l'intérêt en déchiffrant et expliquant la réalité environnementale (paysagère, architecturale, historique). En démontrant sa constance quant à l'application de ces valeurs sur le terrain, le label promet une expérience, vécue et perçue, comparable de lieu en lieu, créant ainsi une attente. Cette garantie d'expérience constitue son empreinte, sa «marque » sur le lieu, car il y laisse bien sa trace. On note que les résultats de terrain de cette étude témoignent que les termes de label et marque représentent le plus souvent une seule et même chose pour les acteurs car « le label comme la marque (branding) sont des gestes sémiotiques, des gestes-signes : retirer, apposer, indiciairiser, aligner, etc. » (Aïm et Lallement, 2016) $)^{2}$.

Qu'on l'appelle mise en marque ou mise en signe du lieu, ce processus visera d'abord à identifier, puis à différencier pour rendre unique. "Vivons l'exception » n'est-il pas d'ailleurs le titre de la newsletter bimestrielle de l'association? C'est par cette exceptionnalisation que la promesse d'expérience est transmise. Tandis que l'expérience est éphémère, la marque est pérenne car elle renforce le souvenir de l'expérience bien après que celle-ci soit révolue dans le temps. Mais la mise en ouvre du label repose aussi sur des activités concrètes : évaluer et conseiller. À travers la Charte de qualité et ses critères, la Commission Qualité évalue le potentiel expérientiel du lieu (pourrait-on déjà l'appeler capital environnemental?) dans chaque village. Elle a également un rôle de conseil auprès des communes pour les soutenir dans leurs actions et leur permettre de les pérenniser. Afin que les communes s'approprient la démarche, un réseau d'échanges de bonnes pratiques

2. Alors que depuis 1991 les PBVF sont une marque déposée à l'INPI bénéficiant de protections juridiques, peu de gens en sont conscients et le mot label reste d'usage courant pour les acteurs interrogés pour qui les enjeux identitaires, paysagers et environnementaux qu'entrainent la « certification », le classement, ne reposent pas sur la différentiation terminologique mais bien sûr la logique du signal que marque et label incarnent semblablement. existe via une plate-forme intranet et une newsletter incarnant, propageant et partageant ainsi les idéaux de la marque, ses valeurs environnementales.

\section{RÉCEPTION DES VALEURS ENVIRONNEMENTALES VÉHICULÉES PAR LE LABEL}

\section{Marque et valeurs environnementales : une émulation?}

En amont, le management de la marque repose sur le désir d'élaborer un portfolio représentatif de lieux qui incarnent ses valeurs environnementales. Quels sont les résultats? Que peut-on dire sur la performativité de la mise en marque? Le label des PBVF est désormais reconnu par un public de plus en plus vaste et exigeant (Abrioux et al., 2016). Il a pour effet de valoriser le village et souvent aboutit à l'internalisation de la marque dans les identités locales quand les habitants se l'approprient et le collectivisent dans leur relation avec le lieu. «Nous sommes un PBVF » et non pas «je vis dans un $P B V F »$. Simultanément, le label stimule une loyauté envers des lieux "marqués » (dans tous les sens du terme) organisés en réseau, y compris de la part d'habitants de communes labellisées qui visitent volontiers d'autres $\mathrm{PBVF}$ « pour voir ce qu'ils ont par rapport à nous ». Le label crée à la fois une attente expérientielle et forme un sens de l'esthétique environnementale en véhiculant ses valeurs. Par exemple, le soigné des abords du village (le premier contact, la rencontre), la valeur architecturale (le repos), et le tracé des cheminements (le mouvement) font écho aux réflexions de Seamon (1979).

Le label peut susciter dans certaines régions un esprit de compétition intercommunale et quelques fois au sein d'une même intercommunalité entre villages labellisés et ceux aspirant au label mais ne possédant pas les qualités requises. Il serait plus juste de dire " pas encore ». Car dans certains villages, tout sera mis en œuvre pour que le lieu vienne à mieux correspondre aux canons du label, dans une démarche inversée. De même, afin de conserver leur appartenance au réseau, les villages labellisés doivent respecter dans leur politique de développement local les engagements pris par rapport au label : "On n'est pas un PBV à vie, le label n'est pas définitif, au contraire, on le voit comme un commen- 
cement» (maire de commune nouvellement labellisée, 2012). Chaque village labellisé, ou « classé », sera ré-inspecté tous les six ans pour rendre compte de la mise en œuvre des engagements par rapport à la Charte de qualité. Le nombre de villages exclus en plus de 30 ans reste très minime et des villages auront choisi de se retirer d'eux-mêmes ne pouvant ou ne voulant pas prendre en compte les avertissements de l'association. Aujourd'hui, celleci fait cependant face à une situation inédite : deux recours déposés en interne par des villages déchus du label après ré-expertise. "Il est probable que nous allons vers une sélection plus forte, avec des déclassements potentiels à la clé, car la tendance est à durcir les critères. [...] Il faudra se pencher un jour sur les villages de première génération qui pour certains ne correspondent plus aux critères, mais qui sont là depuis le début et à qui on doit quelque chose. Nous y sommes sensibles. Cela sera très difficile. Dans un premier temps notre rôle est celui d'accompagnement et de conseil » (membre Commission Qualité, 2014). Ainsi, si la marque représente bien le lieu, il s'ensuit que le lieu cherche à correspondre à la marque. C'est-à-dire qu'en typifiant ou «normant » le village par des critères concrets et fixes, le label encourt le risque de figer l'image du lieu, et par là-même également figer le lieu. C'est face à l'effet normatif de la labellisation que le consensus environnemental peut être incertain.

\section{Représentation et identités environnementales}

\section{Valeurs personnelles ou partagées}

Développement, préservation, et pédagogie représentent les trois axes de la mission de l'association qu'elle résume en quelques mots : " se connaître, se faire connaître, se faire reconnaître », traduisant ainsi la nécessité d’introspection identitaire, le désir de partage et la quête de reconnaissance par l'extérieur. La question de représentation est un point important qui émerge des dynamiques paysagères et environnementales engendrées et véhiculées par la marque. La vision environnementale avancée est-elle représentative, inclusive et participative? L'identité environnementale nourrie par le label est-elle partagée? Qui choisit, impose, et véhicule cette vision? Et par quels moyens? Une bureaucra- tisation de l'authenticité ne se met-elle pas en place sous l'action d'une creative class composée de ce que Zukin (1995), dans le contexte urbain, a appelé des place entrepreneurs? Le processus intervient-il par la base, comme il l'est annoncé dans la promesse du label, c'est-à-dire par les maires et les habitants au niveau local, ou bien par le haut? Est-il bien nécessaire d'avoir des experts, quelquefois extérieurs au lieu, ou bien un heritage from below (le patrimoine par la base) (Robertson, 2012) est-il la meilleure voie à adopter pour une représentation plus juste? Observe-t-on une cristallisation de la culture et du lieu, un manque de fluidité culturelle conséquence d'une raideur structurelle et institutionnelle ou émanant d'une demande homogénéisée consommatrice de campagnes? Autant de questionnements que la labellisation entraîne.

$\mathrm{Si}$, comme on le verra, la représentation environnementale et paysagère engendrée par le label ne fait pas toujours consensus, néanmoins l'enquête de terrain montre que le label a un effet mobilisateur sur la population locale, voire fédérateur dans certains cas. Car en quelque sorte le label lui ouvre les yeux en la sensibilisant au «beau » tel qu'il est construit à travers les critères. Cependant, si les paysages remarquables et extra-ordinaires le sont bien aussi pour les habitants qui y vivent, c'est souvent pour d'autres raisons que celles qui attirent les visiteurs consommateurs de patrimoine. Ils y incorporent d'autres valeurs, personnelles et introspectives.

"C'est dans la plaine que ma famille avait des chevaux avant."

«Nous, on a toujours habité dans ces montagnes. C'est pas toujours facile mais on ne saurait pas vivre autrement. »

"Bien sûr, c'est beau, pour tout le monde, mais pour moi c'est juste mon village. »

"Les monuments classés, c'est important qu'on les reconnaisse, mais avec ça on passe à côté du vrai village. »

"Il y a un village de tous les jours derrière les monuments classés, il ne faut pas l'oublier ou bien nous devenons un musée sans avenir, avec juste le passé. »

Des habitants de villages classés appréhendent le label comme un mécanisme heureux qui conforte leur identité face à des mutations économiques et 
socio-culturelles déstabilisantes. Ils font souvent preuve d'une identité environnementale forte liée au milieu rural de façon très physique, jusqu'à la respiration. Voici quelques exemples de ces éléments identitaires ancrés dans leur environnement :

- Identification à un passé ocrier (figure 9 planche VII).

«On respire jaune depuis toujours. C'est l'ocre. Mais maintenant économiquement c'est du passé... Ça reste dans les souvenirs... Ça serait bien si on pouvait en faire quelque chose, je crois qu'on essaye. »

"L'ocre... c'est notre passé, mais on ne peut y échapper quand on est perché dessus. »

- Construction identitaire et sociale par l'habitat troglodytique (figure 10 - planche VII).

"Nous les Troglos, notre identité c'est la roche; en bas, pour les autres, c'est la rivière."

"En haut c'est nous les Troglos, avant on était les plus riches... en bas ceux de l'eau. Aujourd'hui c'est l'inverse car en bas ce sont les résidences secondaires. Il faut vraiment vouloir y vivre dans un troglo. »

- Attachement viscéral à la pierre (figure 11 planche VIII)

"Ce qui me manque le plus quand je quitte mon village, c'est la pierre... Elle est partout, elle sert à tout, on la creuse, on marche dessus, on vit dedans, on s'y assoit, elle est là pour nous. Partout. »

"Elle est dans mes entrailles... J'ai besoin de la voir au plus profond de moi. C'est physique. »

- D'autres éléments :

"Nous sommes le peuple du tuffeau. »

"C'est la Confluence qui nous a définis."

"La vue sur la plaine du Bourbonnais de ma fenêtre, c'est assez pour me sentir chez moi. »

"Les champs de lavande à l'entrée du village, quand je commence à les apercevoir, je sais que je suis presque arrivé chez moi. »

"Les vignes, ce qui en reste, pas grand-chose, mais ça reste notre histoire et c'est en nous. »

"Ici c'est l'ardoise, on n'y fait plus attention tellement c'est évident dans notre environnement. »

Que les mots soient simples ou elliptiques, ils traduisent pourtant plus que le simple constat d'appartenance au groupe villageois. Ils révèlent un ressenti profond, relevant même d'une totémisation.
Ainsi, l'identification à un élément naturel ou une ressource produite à partir d'un élément naturel (la pierre, l'ocre, la vigne...) est de l'ordre de l'essence et renforce le sentiment de filiation avec ceux qui les ont précédés - les ancêtres qui l'ont connu ou produit- et les distinguent de ceux qui ne peuvent s'attribuer la même ascendance. Sensibilité et connaissance environnementales sont manifestes dans ces paroles qui expriment la relation entre un environnement concret et le « chez-soi » ainsi que la connexion à un passé révolu où les activités rurales donnaient à l'environnement une plus grande importance dans le quotidien. Ce qui n'a pas changé, c'est le lien viscéral avec l'environnement. Ainsi, «l'environnement prolonge le corps, et le corps prolonge l'environnement» (Berque citant Kinji, 2000).

Chez certains habitants, le souvenir de cette connexion à l'environnement dans ses dimensions les plus concrètes est souvent teinté de nostalgie et d'un désir de redécouverte. Par exemple, la prise de conscience de l'eau, la pierre, la lauze, l'ardoise, l'ocre renforce la notion d'identité ancrée dans le local, l'attachement au lieu, l'identité individuelle ou collective. C'est cette conscientisation qui engendre un capital mémoriel, lequel peut être conscient ou inconscient. La « recosmisation » des campagnes serait un désir de nos sociétés (Berque, 2000). Cette « demande de cosmicité » réclamerait « des campagnes qui aient l'air de campagnes, animées par de vrais agriculteurs, et respectant la biosphère » (id.). Dans les villages classés, c'est pourtant le déclin de l'agriculture locale qui a précipité la réorientation de la politique de développement local. C'est dans les ruines des fermes que les PBVF peuvent (re)-naître. Les ruines sont même nécessaires, pour rejoindre J.B. Jackson (1980) : «Un village, ce sont nos belles maisons que le Moyen-Âge nous a léguées, et cet écrin de verdure tout autour. Mais ce sont aussi les gens qui y vivent et qui ensemble maintiennent la vie du village. Tout ça n'est rien sans nous... même s'il a fallu qu'on s'adapte à la nouvelle donne... sans agriculture... Le label nous donne des moyens » (un maire, 2012). La démarche identitaire et sociale du label reste donc forte. Elle s'ancre dans le paysage (l'environnement) et l'appréhension du lieu. Elle s'inscrit dans un désir de continuité matérielle et immatérielle et ce serait une vision réductrice de n'y voir qu'une tentative de marchandisation du local par le tourisme. 


\section{Quand les valeurs ne sont pas partagées}

Pourtant, dans certains villages, on constate parfois une dérive commerciale en décalage avec le lieu, son histoire et sa mémoire, particulièrement dans les régions très touristifiées, et ceci en dépit de la vigilance de l'association et des maires et de la prise de conscience de nombreux habitants. «On n'autorise pas que notre logo soit placé sur des objets commerciaux. On ne veut pas de babioles avec notre logo, des stylos ou des casquettes. Le cadre de l'utilisation du logo est très strict. Quelquefois les gens pensent bien faire, mais ils ne comprennent peut-être pas complètement l'esprit et la démarche de l'association. Il faut les éduquer. C'est aussi là que nous agissons et en cela que nous pouvons aider nos élus » (PBVF, 2013).

La simple couleur des volets revient comme autre point contentieux. La palette conseillée est parfois perçue comme une restriction excessive et une entrave à la liberté de vivre et de créer son espace de vie, plutôt qu'une protection nécessaire du patrimoine. Parfois les couleurs proposées s'opposent à la mémoire individuelle du lieu : «Mes grands-parents ont habité ici toute leur vie, les volets n'ont jamais été de cette nouvelle couleur. C'est pas vraiment traditionnel en fait... c'est pas comme ça que je me souviens de la maison quand j'y venais petit... J'aimerais bien pour mes enfants que ça soit "comme avant", comme je le voyais "moi" à leur âge. Mais eux et ma femme ils aiment bien, ils trouvent ça plutôt chouette, alors... c'est plus nous qui décidons... et ce n'est que de la peinture! " (Habitant permanent de « retour », 2012). Les obligations esthétiques peuvent donc être ressenties comme une altération d'un capital mémoriel. Ailleurs, des volets de couleur vive en rupture avec l'harmonie chromatique d'un village entraînent des discordes, ce choix délibéré perçu comme un mode de résistance : «Ils font ce qui leur chante " (figure 12 - planche VIII).

De même, dans plusieurs villages, des habitants expriment des réserves quant à l'installation d'expositions temporaires, notamment d'art contemporain, perçues comme déconnectées de l'essence du lieu et non-représentatives de leurs préoccupations immédiates et de leur identité. Leurs mots traduisent ce qu'ils vivent comme une dépossession :

"Cela ne nous représente pas et ne représente rien pour nous. Ça pourrait être n'importe où, ici, à Paris, ou à Singapour. »
«Ça ne me plaît pas parce que surtout je ne comprends pas ce que c'est et pourquoi c'est venu là. On reste extérieur et pourtant c'est chez nous. Enfin, ça devrait être chez nous. Et c'est sûr qu'on a dî payer pour ça.»

«Les gens, ils ne captent pas. Ça leur semble tellement étranger. Ça prend de la place pour attirer des gens qui viennent pour ça mais qui se fichent pas mal du village. Et même les artistes, est-ce qu'ils sont même venus se promener? Ils aiment se produire dans un "Plus beau village", et c'est tout. "

"À quoi ça rime? Il nous faudrait plutôt un dépôt pain ou une pharmacie. C'est sûr que le public visé c'est pas nous. »

Enfin, la valeur environnementale étant souvent associée à la valeur écologique, il convient de considérer l'appréhension du développement durable au sein des communes labellisées. Pour Garat et al. (2005) la patrimonialisation et le développement durable émanent de la même logique de transmission vers des générations futures. Les deux approches s'inscrivent également dans une même mission pédagogique. Dans la démarche de l'association, le développement durable apparaît plus en filigrane que de front (Ducros, 2016), car la Charte de qualité implicitement limite le développement des énergies renouvelables dans le paysage, notamment par la réticence esthétique vis-àvis des panneaux solaires sur les toitures : « Ce qui crée l'émotion, c'est l'harmonie. Alors, un toit par ci comme ça, un autre ailleurs comme ça... Ça ne veut pas dire que nous sommes contre. Un jour le progrès de la technologie nous permettra d'allier nos exigences par rapport au bâti avec des techniques plus durables. Des tuiles solaires ou autres. Mais pour l'instant c'est la ligne que nous avons choisie et on s'y tient. » De même, un maire explique la tension entre capital esthétique et les infrastructures nécessaires à la production d'énergies renouvelables : "Il ne faut pas qu'on laisse s'installer n'importe quoi n'importe où. On ne vient pas en Provence pour voir des toits en panneaux solaires... Les gens viennent pour la tuile, la couleur, les reflets de la lumière changeante, ils s'y retrouvent et nous aussi. " La dimension écologique reste ambiguë car elle se heurte à la recherche de l'émotion dans le paysage. Elle est entravée par la représentation du lieu dans l'imaginaire collectif et la qualité identitaire renforcée par les recomman- 
dations du label quant à l'utilisation de matériaux, formes, couleurs et volumes. Ces quelques points illustrent que les normes promues par la Charte de qualité et intégrées dans les politiques locales de développement peuvent être reçues comme des obstacles pour la mise en œuvre d'autres conceptions paysagères basées sur d'autres valeurs.

\section{Hégémonie des valeurs environnementales véhiculées par le label au-delà des PBVF ?}

L'exportation du modèle Plus beaux villages pose la question du pouvoir d'homogénéisation des valeurs environnementales véhiculées par la Charte de qualité. Ces valeurs possèdent-elles un potentiel d'uniformisation culturelle, suivant un processus hégémonique souvent redouté par les altermondialistes ? La diffusion du modèle s'est affirmée depuis le nouveau millénium. Quatorze associations ont été créées à ce jour dans d'autres nations à l'image de l'association française et la liste des pays intéressés est en constante expansion, de la Chine au Canada en passant par des pays du Moyen-Orient. Tandis que c'est autour du lien francophone ou de proximité que s'établissent les premières trajectoires de diffusion, des foyers de diffusion décentralisés opèrent désormais (Ducros, 2014). Par exemple, c'est en grande partie sous l'influence de l'association italienne I Borghi Più Belli d'Italia que la Roumanie a créé son réseau. C'est aussi par l'Italie que se renforce un sous-réseau en région «Méditerranéenne ». En Asie, c’est le Japon qui irradie le modèle vers la Corée du Sud. Le capital social des acteurs en réseau s'impliquant dans la diffusion dépasse les frontières du local, créant un translocalisme au sein duquel l'identité du rural est partagée et les idées de préservation et développement circulent. La Charte de qualité devient un modèle qui s'adapte aux possibilités locales et valide la démarche. "Une Charte de qualité à la française est une base essentielle pour construire notre propre réseau et convaincre les sceptiques que notre démarche est sérieuse » (président de PBV à l'étranger, 2012). Une coopération officielle entre les réseaux les plus établis est entérinée en 2012 avec la création d'une Fédération internationale des plus beaux villages de la Terre.
Comme le label devient lui-même patrimoine, ce « patrimoine-label » devient capital puisqu'il en présente des caractéristiques princeps. Il est transmissible aux générations suivantes, et même transformable, par exemple en capital économique en soutenant l'intérêt touristique. "Ce label, on va le transmettre à la nowvelle génération. On espère qu'ils en seront conscients et qu'ils en prendront soin, car ça a été du travail. Ce n'était pas gagné d'avance» (maire de commune récemment labellisée, 2013). "Le village fait partie des premiers labellisés. On a fait l'histoire du label en quelque sorte. Et le label fait partie de notre histoire aussi. Ce n'était pas facile an début... on s'est quand même battu. Ce label, on doit le garder, absolument, et le transmettre. C'est un atout pour l'avenir » (maire de village première génération, 2011). Ce désormais « capital-label », opérateur de la ressource territoriale, transcende les frontières par la mise en réseau de communautés partageant les mêmes valeurs environnementales et s'appuyant les unes sur les autres dans l'expérience et l'imaginaire du rural que (re)produit la marque.

\section{Conclusion : UN CAPITAL RELATIONNEL, TRANSPOSABLE ET TRANSLOCAL BASÉ SUR LA VILLAGITÉ}

Cet article aborde la construction, réception, conscientisation collective, et utilisation d'un système de valeurs selon lesquelles des acteurs de la patrimonialisation territoriale s'investissent dans leur environnement par le biais d'un label, celui de l'Association des plus beaux villages de France. Sa Charte de qualité établit des critères de sélection qui englobent un patrimoine à préserver ainsi qu'une mémoire historique et spatiale reposant sur un mode de vie rural, fut-il passé. Il a eu un double effet, l'un élitiste, ne reconnaissant qu'un nombre restreint de communes, l'autre normatif, définissant un espace géographique - le village - dont tous les éléments sont constitutifs. Ainsi, le jardinet comme la porte sculptée ou les étroites ruelles, le châtelain, comme l'artisan ou le retraité participent de l'ensemble idéel sans attribution nominale et dans une communauté de destin.

Créant un ensemble de valeurs liées à l'environnement, la labellisation des territoires influence les rapports société-milieu et paysage-patrimoine. Les constituants du label reposent également sur 
la construction synchronique de la valeur patrimoniale des lieux. Celle-ci est fortement liée à d'autres valeurs - identitaires, symboliques et mémorielles qu'un attachement affectif au lieu suscite à travers l'expérience physique, multi-sensorielle et esthétique dans ce lieu. Suivant un processus de «imagineering ${ }^{3}$ », ou « imaginerie », l'environnement et sa représentation sont ré-imaginés et (re)créés. Par un effet de feedback loops la labellisation construit et propage ainsi de nouvelles valeurs standardisées basées sur l'idéalisation du local et une "typicité » ancrée dans la mémoire et l'histoire du lieu bien que parfois ré-inventée. Il en résulte un paradoxe : une standardisation de la spécificité paysagère et territoriale, au cœur de laquelle patrimoine et environnement se conjuguent.

L'étude démontre que la labellisation patrimoniale peut non seulement s'inscrire dans le champ théorique d'un capital environnemental, mais qu'elle aiderait aussi à en éclaircir les contours. Le cas spécifique en France rurale apporte des réponses pour comprendre certains de ses enjeux, par exemple la question de savoir s'il serait partagé, privé ou public, ou même encore relationnel, i.e. source, agent et résultat d'un système de relations. Le label des PBVF, plus qu'un avatar de la ruralité, devient acteur de la ressource territoriale à travers sa marque relationnelle (relational branding) qui lie dans un même réseau des lieux spatialement discontinus soumis aux mêmes exigences esthétiques et patrimoniales basées sur des valeurs environnementales partagées. En mettant en exergue la réception et la diffusion des valeurs produites par le processus de labellisation, l'étude éclaire la relation entre paysages ruraux labellisés et un champ social de l'environnement. La charte de qualité, sur laquelle repose l'action de l'association, reconnaît, renforce et promeut de multiples composantes paysagères auxquelles viennent s'ajouter la qualité symbolique et mémorielle du lieu de vie et la qualité identitaire produite et reproduite dans et par le lieu. Ainsi, la Charte, en produisant et véhiculant des valeurs multiples mais précises, se transforme en recueil de valeurs, délimitant ce champ de l'environnement, à partir duquel peut alors s'élaborer un capital environnemental. La démarche des acteurs illustre donc une façon dont la labellisation patrimoniale des territoires s'inscrit

3. Néologisme anglophone fusionnant image, invention, et fabrication (imagine + engineering) du lieu. dans un concept de capital environnemental, participant à son contenu et contribuant à en définir un domaine d'application. Par le label, l'environnement acquiert une valeur symbolique qui donne prestige au lieu. Le capital environnemental se sublime alors en capital symbolique qui s'appuie sur une même rhétorique identitaire, construite sur des pratiques, une éthologie et un imaginaire communs que l'on appellera «villagité ».

Le mode de vie rural, idéalisé au-delà des spécificités locales et cristallisé par le label, devient une composante du champ environnemental, constituant ainsi un atout pour l'émergence d'un capital environnemental dont la transposabilité est démontrée à travers l'expérience décentralisée de la Fédération internationale qui en fait un capital translocal en adaptant un modèle français de re-paysement à d'autres milieux ruraux : "Le décor change mais nous sommes tous les mêmes, tous des ruraux... " (Président d'association étrangère, 2012). Il apparaît également que les échanges translocaux et le transfert d'une creative knowledge stimulent la conversion du capital social des acteurs en un capital environnemental qui n'est plus désormais attaché à un territoire mais à des valeurs communes ancrées dans la nouvelle ruralité. Ce capital environnemental serait donc avant tout relationnel, opérant dans un enchevêtrement d'échelles, de vecteurs, et d'acteurs humains et non-humains. Même si ce capital naît d'une entente entre les acteurs locaux (à travers la décision du conseil municipal obligatoire pour postuler au label), le consensus reste cependant fragile dans les communes où les valeurs ne sont pas partagées et où l'on constate un sentiment de dépossession du lieu par le label, en fait un dé-paysement des campagnes pour certains habitants.

L'appréhension de l'environnement, sensu lato - individuelle par chaque villageois, collective pour l'association - révèle une diversité de réflexions qui montre qu'un «capital environnemental » est bien appréhendé, sans être formulé de façon analytique. Tout se passe comme si celui-ci consistait en un emboîtement de capitaux distincts, quoique reliés entre eux : capital émotionnel, capital mémoriel, capital identitaire... Tel des boîtes gigognes à la façon des matriochkas dont la plus grosse ne prend son sens qu'en englobant les autres. Ou alors, le capital environnemental, par analogie avec la notion mathématique, serait un « ensemble flou » aux 
limites rendues imprécises par le fait qu'il contient entièrement certains attributs mais partiellement d'autres. Ou bien irait-on jusqu'à l'assimiler à la notion de «signifiant vide » qui absorbe et véhicule les significations stipulées par ceux qui le définissent et dont les interprétations peuvent être multiples et même contradictoires?

\section{Remerciements}

Merci à l'équipe de GEOLAB à Limoges pour son accueil et soutien, à l'Association des plus beaux villages de France pour sa généreuse assistance, aux maires et villageois qui m'ont ouvert leurs portes, à la National Science Foundation pour son financement (grant\# 1202703) et aux relecteurs anonymes pour leurs recommandations.

\section{Bibliographie}

Abrioux F., Kaswengi J., Welté J.-B., 2016. Usage et appropriation des labels : comment faire sens avec ces signes?, JurisArt, no 4l, p. 36-38.

Aїм O., Lallement E., 2016. Labels du patrimoine : une nouvelle dialectique entre art et économie?, JurisArt, $\mathrm{n}^{\circ} 41$, p. 23-26.

Alaux F., de Crécy H., Houplain L., 2009. Logorama, Studio H5, 16 min.

Berleant A., 1997. Living in the landscape, towards an aesthetics of environment, Lawrence, University Press of Kansas, 176 p.

Berque A., 2000. Ecoumène: Introduction à l'étude des milieux humains, Paris, Belin, 272 p.

Bonerandi E., 2005. Le recours au patrimoine, modèle culturel pour le territoire, Géocarrefour, nº 80, vol. 2, [https://geocarrefour.revues.org/991?lang=en].

Bouisset C., Degrémont I., 2013. La patrimonialisation de la nature : un processus en renouvellement, L'Espace géographique, no 42, p. 193-199.

Bourdieu P., 1979. La distinction : critique sociale du jugement, Paris, Minuit, $670 \mathrm{p}$.

Bourdieu P., 2000. Propos sur le champ politique, Lyon, Presses universitaires de Lyon, $111 \mathrm{p}$.

Cailly L., Dodier R., 2007. La diversité des modes d'habiter des espaces périurbains dans les villes intermédiaires : différenciations sociales, démographiques et de genre, Norois, vol. 205, p. 67-80.

Capello R., Caragliu A., Nijkamp P., 2011 . Territorial capital and regional growth: Increasing returns in knowledge use, Tijdschrift voor Economisch en Sociale Geografie, $\mathrm{n}^{\circ}$ 102, vol. 4 , p. 385-405.
Charbonnier P., 2015. La fin d'un grand partage. Nature et société, de Durkheim à Descola, Paris, CNRS, 314 p.

Ducros H., 2014. Protect, promote, develop : Rural tourism and village patrimonialization, in Dashper K. (ed.), Rural Tourism: An International Perspective, Cambridge Scholars Publishing, p. 59-78.

Ducros H., 2017. Confronting sustainable development in two rural heritage valorization models, Journal of sustainable tourism, $\mathrm{n}^{\circ} 25$, vol. 3, p. 327-343.

Filloz V., Соцомв V., 2011 . De l'étiquette à la marque : la labellisation comme unificateur des projets territoriaux? Développement durable et territoires, $\mathrm{n}^{\circ} 2$, vol. 2, [https:// developpementdurable.revues.org/8998?lang=en].

Fournier M. (ed.), 2014. Labellisation et mise en marque des territoires, Clermont-Ferrand, Presses universitaires BlaisePascal, $634 \mathrm{p}$.

Garat I., Gravari-Barbas M., Veschambre V., 2005. Préservation du patrimoine et développement durable : une tautologie? Les cas de Nantes et Angers, Développement Durable et Territoires, Dossier 4, [https://developpementdurable.revues. org/4913].

GEOLAB, 2015. Appel à contribution pour le colloque Capital environnemental : Représentations, pratiques, dominations, appropriations spatiales, Université de Limoges 18-21 novembre, $4 \mathrm{p}$.

Guyot S., Richard F., 2009. Les fronts écologiques - Une clef de lecture socio-territoriale des enjeux environnementaux?, L'Espace Politique, $\mathrm{n}^{\circ}$ 9, vol. 3, [https://espacepolitique. revues.org/1422?lang=en].

Håkansson N.T., Widgren M. (eds.), 2014. Landesque capital: The historical ecology of enduring landscape modifications, Left Coast Press, 281 p.

Hartog F., 2003. Régimes d'historicité. Présentisme et expériences $d u$ temps, Paris, Seuil, $272 \mathrm{p}$.

Heinich N., 2009. La fabrique du patrimoine. « De la cathédrale à la petite cuillère », Paris, Éditions MSH, 288 p.

Husson J.-P., 2008. Envies de campagnes, les territoires ruraux français, Paris, Ellipses, 208 p.

JACKSON J.B., 1980. The necessity for ruins, and other topics, Amherst, University of Massachussetts Press, 136 p.

Landel P-A., Senil N., 2009. Patrimoine et territoire, les nouvelles ressources $d u$ développement, Développement durable et territoires (consulté 13 juillet 2009), [https://developpementdurable.revues.org/7563].

LARRue C., 2017. Les modalités institutionnelles d'une nouvelle ruralité : « de l'environnement au rural »?, in Hamman P. (dir.), Ruralité, nature et environnement, entre savoirs et imaginaires, Toulouse, Erès, p. 455-484.

Lazzarotti O., 2011. Patrimoine et tourisme, histoires, lieux, acteurs, enjeux, Paris, Belin, 304 p.

Lowenthal D., 2015. The Past is a Foreign Country-Revisited, Cambridge Press, 676 p.

Lowenthal D., Riel M., 1972. The nature of perceived and imagined environments, Environment and Behavior, $\mathrm{n}^{\circ} 4$, vol. 2, p. 189-207. 
MeINIG D.W., 1979. The interpretation of everyday landscapes: Geographical essays, New York, Oxford University Press, $272 \mathrm{p}$.

Neveu E., 2013. Les sciences sociales doivent-elles accumuler les capitaux?, Revue française de science politique, $\mathrm{n}^{\circ}$ 63, vol. 2, p. 337-358.

Perucca G., 2014. The Role of Territorial Capital in Local Economic Growth: Evidence from Italy, European Planning Studies, $\mathrm{n}^{\circ}$ 22, vol. 3, p. 537-562.

Patton M.Q., 2002. Qualitative research and evaluation methods, $3^{\text {rd }}$ ed., New York, Sage, 688 p.

Pike A., 2015. Origination: The Geographies of Brands and Branding, Chichester, Wiley, 248 p.

Plus Beaux Villages de France, 2016. Point.com, nº 42, 4 p.

Plus Beaux Villages de France. La sélection des villages: instruction des demandes de classement, [http://www.lesplus-beaux-villages-de-france.org/files/ier_d_instruction_des_ demandes_de_classement.pdf], 4 p.

Poulot D., 2006. Une histoire du patrimoine en occident, Paris, PUF, 196 p.
Relph E., 1976. Place and placelessness, London, Pion, 156 p. Richard F., 2010. Capital environnemental et recompositions socio-spatiales du péri-urbain : les leçons de l'expérience britannique, in Laaouane M., Aкdim B., Aménagement périurbain. Processus, enjeux, risques et perspectives, Fes, Morocco, p. 117-128.

Rickly-Boyd J., Knusden D., Braverman L., MetroRoland M., 2014. Tourism, performance, and place: A geographic perspective, Farnham, Ashgate, 196 p.

Robertson I. (ed.), 2012. Heritage from Below, Farnham, Ashgate, $264 \mathrm{p}$.

SEAmon D., 1979. A Geography of the Lifeworld: Movement, Rest and Encounter, London, Croom Helm, 232 p.

Tuan Y.F., 1974. Space and place: The perspective of experience, Minneapolis, University of Minnesota Press, 248 p.

Wood N., 1999. Vectors of memory: legacies of trauma in postwar Europe, Berg, 224 p.

Zukin S., 1995. The Culture of Cities, Cambridge, Blackwell, $322 \mathrm{p}$. 